\title{
Intelligence and gelotophobia: The relations of self-estimated and psychometrically measured intelligence to the fear of being laughed at
}

\author{
RENÉ T. PROYER and WILLIBALD RUCH
}

\section{Abstract}

The present article examines the relation between the fear of being laughed at (gelotophobia) and intellectual abilities (verbal, numeric, and spatial intelligence, memory and reasoning, vocabulary, and attention) and the self-estimation of one's own abilities. In a first study, $N=167$ participants completed ability tests along with a subjective measure for gelotophobia. The results indicate that gelotophobia and intellectual abilities exist independently from each other. These results were replicated in a second study $(N=177)$ with an independently collected data set. In this study the participants also completed a form for the self-estimation of their own abilities. Though there was a tendency for lower self-estimations of their own abilities, the mean scores were not significantly different among groups of nongelotophobes and participants with borderline, slight, and pronounced fear of being laughed at. However, the differences between psychometrically measured and self-estimated abilities showed that gelotophobes have a lower self-estimation of their abilities regarding general intelligence, vocabulary, and attention. Taken together the studies show that gelotophobia is not related to intelligence but that gelotophobes tend to have lower selfestimations of their own abilities and underestimate their true ability (i.e., psychometrically measured) by 6 IQ-points. The general pattern of low self-estimations of abilities in gelotophobes is discussed and whether this might be a useful starting point for the development of treatments for gelotophobia.

Keywords: Gelotophobia; humor; intelligence; laughter; self-estimated intelligence. 


\section{Studying the fear of being laughed at and ability}

A reasonable effort has been spent on the exploration of the experiential world of gelotophobes and their convictions and beliefs (see this issue; Platt 2008; Ruch and Proyer 2008a, 2008b). Still, little is known about the abilities of gelotophobes. At least two different views on this relation are likely. First, one might argue that people with low (intellectual) abilities fear being laughed at for good reasons - presumably they experience being laughed at more often because they cannot keep up with others in many different situations; i.e., they say and do silly things and get laughed at. Thus, they fear what they truly experience in their everyday life. Second, one might argue that gelotophobes only think they are ridiculous and inferior compared to others, but that they do not differ (from others) regarding their actual abilities. In this case, their fear is based on imagined inferiorities only. For example, there is empirical evidence that the gelotophobes' self-estimated virtuousness is lower than observer reports on their actual strengths (Proyer and Ruch this issue). This leads to a similar line of argument as suggested by Proyer et al. (this issue). In their study, gelotophobes did not remember events of being laughed at (in a given time span of twelve months) more frequently than nongelotophobes. Nevertheless, they feared such events (of having been laughed at) to a (far) higher degree.

Intelligence seems to be best suited for a study on the relation between gelotophobes' self-reported and psychometrically measured ability. Many reliable and valid measures of different aspects of intelligence are available and provide a solid foundation for the examination of putative differences in the intelligence of gelotophobes. Models of intelligence differ mainly with respect to whether they refer to one general factor of intelligence (g-factor, "general mental ability"; e.g., Galton, McKeen Cattell, or Spearman), to two factors (general intelligence and specific factors; Spearman), or to multiple factors (e.g., Guilford's three dimensional 'structure of intellect' model or the seven primary mental abilities put forth by Thurstone). Other theorists proposed hierarchical models of intelligence. For example, Vernon described, next to a general factor of intelligence, so-called major group factors (verbal-educational and practical abilities), minor group factors (e.g., verbal, number, spatial etc.), and specific factors. Cattell suggested distinguishing among fluid (reasoning ability) and crystallized (acquired knowledge over one's life-span) intelligence that are second-order factors of the g-factor (see Cooper 2002 for 
an overview). For the present studies we integrate information on the general ability of a person (as a total score out of specific abilities) and his/her specific abilities (verbal, numeric, spatial intelligence, attention, memory), and crystallized (vocabulary) and fluid intelligence (reasoning).

A study by Ruch et al. (this issue) provides the first hints on the possible relations between ability and gelotophobia. One of the results of a comprehensive study on the humor of gelotophobes was that the ability to create humor (as measured with the Cartoon Punch line Production Test by Köhler and Ruch 1993, 1996) was not related to gelotophobia. This was interesting because the initial hypothesis was that gelotophobes would score lower because they evaluate their humor ability negatively themselves. For example, they describe their humor style as inept (i.e., lacking skill and confidence in dealing with humor). Typically gelotophobes would feel uneasy in the company of humorous people. They feel inferior compared to them and are convinced they cannot keep up with them. In the performance tasks of the CPPT, however, there is no relation between gelotophobia and humor production and no relation to the wit of the created punch lines (assessed by peer ratings). Overall, the study shows that having the ability to create funny punch lines and the fear of being laughed at exist independently from each other. There are gelotophobes lacking humor construction ability but there are also gelotophobes that are good in creating humor. Based on this study the question emerges whether there is a typical pattern for gelotophobes and their under-estimations of their own abilities. A study aimed at answering this question entails ideally both, the psychometrically measured and the selfrated intelligence of a person.

\subsection{The relation between personality and intelligence}

Eysenck (1994) argued that IQ is not related to personality but that the actual performance in an IQ-test is related to personality. Trait anxiety should have a special influence on test scores, but only if the conditions of testing induces it (state anxiety; e.g., stressful situations). Ackerman and Heggestad (1997) performed a meta-analysis on the relation between intelligence and personality. A few of their findings should be highlighted. Among other results, they found that personality traits that are related to Neuroticism and Psychoticism tend to be related negatively to ability, whereas personality traits related to Extraversion tend to be positively 
related to intelligence. Furthermore, they report positive correlations with the typical intellectual engagement (TIE) of the person and Openness to Experience. Though they found a pattern of typical relations, they were moderate in size.

However, different variables might have an impact on the personalityintelligence relation. For example, Moutafi et al. (2006) showed that the relation between Neuroticism and intelligence is mediated by test anxiety (see also Hopko et al. 2005; Meijer and Oostdam 2007). The cognitive ability of the persons itself plays an important role as well (Perkins and Corr 2006). Furthermore, the positive Extraversion-intelligence relation is not found in all studies. In fact, there are also studies that point in the opposite direction (see Moutafi et al. 2006 for an overview).

In one of the studies reported in the present paper, there is interest in the participants' self-estimations of their abilities. How good are people in estimating their own abilities? Chamorro-Premuzic et al. (2004) report correlations between $r=.39$ and $r=.49$ between psychometrically measured and self-estimated intelligence; which is slightly higher but in the same direction as in an older meta-analysis by Mabe and West (1982). Furnham (2001) concludes a literature review by stating that there is a positive but only modest correlation between test scores and self-ratings of intelligence. Furthermore, personality seems to have an impact on the judgments as well. For example, there is empirical evidence that emotionally stable persons tend to have higher self-estimates of their intelligence (Furnham and Chamorro-Premuzic 2004; Chamorro-Premuzic et al. 2005). For the present study, it is of interest whether gelotophobes differ in their self-estimated intelligence from non-gelotophobes and whether gelotophobia is related to ability at all.

\subsection{Aims of the present study}

Study 1 is aimed at an examination of the relationship between gelotophobia and intelligence. It is expected that gelotophobes do not differ in their abilities from non-gelotophobes and that the mean scores of gelotophobes and non-gelotophobes in several ability tests will be highly similar. This will be examined for the relation to the sample used in the present study, as well as to the normative sample for tests that assess verbal, numeric, and spatial intelligence. Furthermore, we expect that groups of high-scoring gelotophobes (i.e., pronounced/marked gelotophobia ac- 
cording to Ruch and Proyer 2008a) do not differ from other gelotophobes and non-gelotophobes regarding their abilities and also that the correlations between gelotophobia and intelligence are the same for high and low scorers.

Study 2 is designed to replicate the findings from the first study and to examine the relation between psychometrically measured and selfestimated intelligence. It is expected that gelotophobes have lower selfestimations of their own abilities than non-gelotophobes - and that they underestimate their abilities. By using a difference score between psychometric and self-estimated intelligence, it can be determined whether there are differences in all abilities or whether there are abilities with larger differences than others. Putative differences in the abilities after transforming the raw scores to the IQ based on the normative data in the test handbook can also be examined.

\section{Method}

\subsection{Research participants for Study 1}

The sample consisted of $N=167$ participants $(n=54$ males and $n=113$ females) with an age range from 18 to 77 years $(M=45.28, S D=14.20)$. $1.80 \%$ did not have a school-leaving certificate, $38.32 \%$ completed a vocational education, $11.38 \%$ had a degree from school that qualified for the attendance of a university, $28.74 \%$ were students or had a degree from a school of higher education, and $19.16 \%$ were students or held a degree from university.

\subsection{Research participants for Study 2}

The sample consisted of $N=177$ participants from 19 to 68 years $(n=70$ males and $n=107$ females). Their mean age was $39.58(S D=11.19)$. One of the participants had ground school as highest educational level, $41.81 \%$ completed a vocational education, $6.78 \%$ had a degree from school that qualified for the attendance of a university, $23.16 \%$ were students or had a degree from a school of higher education, and $27.12 \%$ were students or held a degree from university. 


\subsection{Instruments for Study 1}

The Geloph $\langle 15\rangle$ (Ruch and Proyer 2008a) is a 15-item measure for the subjective assessment of gelotophobia. All items are positively keyed and they utilize a four-point answer scale (from $1=$ strongly disagree to $4=$ strongly agree). A sample item is "When they laugh in my presence I get suspicious." The reliability coefficient of the questionnaire was .91 (Cronbach alpha). The GELOPH $\langle 15\rangle$ is the standard instrument used for the assessment of gelotophobia (see this issue).

The Intelligence Structure Test (I-S-T 2000-R; Amthauer et al. 2001) consists of nine subtests, two tests for memory (verbal and spatial), and a general knowledge test. It allows the assessment of fluid as well as crystallized intelligence. For the present study we used subtests for verbal (analogies), numerical (arithmetical tasks), and spatial (cube tasks) intelligence, and memory (for verbal contents). The tests for verbal, numeric, and spatial intelligence were taken together as a total score of intelligence. All tests are speed tests. The I-S-T $2000 \mathrm{R}$ is widely used and well-established in the German speaking countries.

The $d 2$ test of attention (Brickenkamp 1994) is a measure of attention and concentration performance. It consists of 14 rows of 'd's and 'p's. Each is marked with one, two, three, or four apostrophes. The task of the participant is to mark each ' $\mathrm{d}$ ' that shows two apostrophes. The $\mathrm{d} 2$ is a speed test (20 seconds per row). The final score used in the present study is calculated by subtracting the number of wrongly marked letters from the total number of marked letters. The $\mathrm{d} 2$ is widely used in research and practice in the German speaking countries.

The WST (Schmidt and Metzler 1992) is a multiple choice vocabulary test. It consists of 42 rows with six words each. Only one of them is a real word and the other ones are distractors that were chosen because they sound similar. The task of the subject is to mark the meaningful word. The items are rank ordered according to their difficulty. The WST is a power test and one of the standard tests for vocabulary in the German speaking countries.

The Standard Progressive Matrices (SPM; Raven et al. 1998) is a test for the non-verbal assessment of the ability of reasoning. The task of the test taker is to identify one out of a set of six figures that completes a pattern (a diagram or design) that is given on the top of the page. There is only one correct answer for each pattern). It is considered to be a valid measure of general intelligence. The SPM consists of five sets of twelve 
different matrices that are rank ordered according to their difficulty (starting with the easiest matrices). The testing time was limited to 20 minutes.

\subsection{Instruments for Study 2}

The same instruments as in Study I were used; i.e., the GELOPH $\langle 15\rangle$ (Ruch and Proyer 2008a; Cronbach alpha $=.88$ ), four subtests out of the Intelligence Structure test (Amthauer et al. 2001), the $d 2$ test of attention (Brickenkamp 1994), a vocabulary test (Schmidt and Metzler 1992), and the Standard Progressive Matrices (Raven et al. 1998).

2.4.1. Measure for self-estimated intelligence (MSEI; Proyer and Ruch 2008). Participants have to rate their ability on a line from "low" to "high ability" for the domains of verbal, numeric, and spatial intelligence and for memory, reasoning, attention/concentration, and their vocabulary. Each position on the scale ranging from lowest to highest selfestimated ability may be marked (on a scale from $0=$ lowest ability to $100=$ highest ability). A total score was computed out of all selfestimations as a general self-estimated ability score. The single dimensions were explained by a short sentence (e.g., "reasoning: being fast in recognizing connections").

\subsection{Procedure}

The sample of Study 1 consisted of participants in a positive psychologytraining program at the department of psychology at the university of Zurich ("Zurich Strengths Program"). The authors of this study were the initiators and leaders of this program. The participants were recruited via reports in newspapers, pamphlets, and the website of the department. Participants needed to be older than 18 years of age; there were no further restrictions for the participation. All participants filled in questionnaires at home and were tested with the ability tests at the psychology department. The whole procedure took approximately 90 minutes including a short break. All tests were administered in group settings. Psychologists that were specially trained in psychological assessment provided the testing. Two higher-grade students helped with the administrative parts of the testing sessions. The participants first completed the subtests from 
the I-S-T 2000 R test battery, followed by the d2, and the SPM and WST after a short break (10 minutes).

The testing for Study 2 was done independently from Study 1 but under the same conditions. The whole procedure for Study 2 took approximately 95 minutes. Participants were not charged fees for their participation in the program and were not paid for their services. All participants received individual feedback on their results after the program was completed.

\subsection{Results}

Mean scores and standard deviations were computed for all subtests, tests, and a total score for general intelligence that consisted of the subtests for verbal, numeric, and spatial ability of the I-S-T 2000-R. Additionally, a total score was computed for the self-estimations as a measure of general self-estimated ability (Study 2). The skewness and kurtosis of the measures indicated that all scales were normally distributed. Table 1 shows the descriptive statistics of the scores from the two studies.

Table 1 shows that the mean scores were highly similar in the samples from Study 1 and Study 2. However, in a $t$-test for independent samples, the scores for reasoning (SPM) and spatial intelligence were significantly

Table 1. Descriptive statistics for the measures of gelotophobia and intelligence

\begin{tabular}{|c|c|c|c|c|c|c|}
\hline & \multicolumn{2}{|c|}{$\begin{array}{l}\text { Psychometrically } \\
\text { measured (Study 1) }\end{array}$} & \multicolumn{2}{|c|}{$\begin{array}{l}\text { Psychometrically } \\
\text { measured (Study 2) }\end{array}$} & \multicolumn{2}{|c|}{ Self-estimated } \\
\hline & M & SD & M & SD & M & SD \\
\hline Gelotophobia & 1.81 & 0.52 & 1.86 & 0.57 & - & - \\
\hline Verbal & 11.67 & 3.02 & 11.00 & 4.07 & 66.54 & 18.74 \\
\hline Numeric & 12.04 & 4.43 & 12.02 & 4.79 & 52.03 & 24.72 \\
\hline Spatial & 9.69 & 4.12 & 8.61 & 4.18 & 59.53 & 24.02 \\
\hline Memory & 6.82 & 2.34 & 6.51 & 2.79 & 58.16 & 19.65 \\
\hline Total & 33.70 & 8.57 & 31.80 & 9.95 & 69.39 & 18.55 \\
\hline Reasoning & 46.34 & 6.24 & 43.81 & 8.38 & 66.19 & 20.27 \\
\hline Vocabulary & 33.20 & 2.44 & 33.45 & 2.76 & 69.67 & 18.27 \\
\hline Attention & 164.83 & 40.38 & 159.67 & 43.31 & 62.33 & 17.89 \\
\hline
\end{tabular}

$N=142-167$ (study 1 ) and $N=168-174$ (study 2). $M=$ mean, $S D=$ standard deviation. Total $=$ total score of verbal, numeric, and spatial intelligence for psychometrically measured intelligence and a global score of all estimations for the self-estimated intelligence; the highest possible score for the self-estimations in the MSEI is 100. 
higher in Study $1(t(357)=-2.87, p<.01$; and $t(357)=-2.18, p<.05$, respectively). Study 2 allowed for a comparison of psychometrically measured and peer-reported intelligence. The mean scores indicated that the psychometrically tested intelligence (out of the I-S-T $2000 \mathrm{R}$ ) was highest for the numeric intelligence, while verbal intelligence was the highest for the self-estimated abilities.

An ANOVA with four groups (non-gelotophobes, borderline, slight, and pronounced/extreme gelotophobes; see Ruch this issue; Ruch and Proyer 2008a) and the ability tests as dependent variables did not reveal mean score differences among the groups neither for the psychometrically measures intelligence (Study 1 and 2) nor for the self-estimated intelligence (Study 2). None of the mean scores were statistically different from one another. However, the ability mean scores were lowest for the group of highest scoring gelotophobes and probably did not reach statistical significance because of the low number of participants in the highest scoring group (i.e., $n=9$ and $n=5$ in Study 1 and 2, respectively).

The same results emerged when the dependent and independent variables were changed in the analysis. We also used the total score of the subtests from the intelligence test-battery (I-S-T $2000 \mathrm{R}$ ) as an indicator of general intelligence for the further analysis. This score was split into six groups of equal size ranging from lowest to highest intelligence. We computed an ANOVA and used gelotophobia as a dependent variable with the six intelligence groups as classification variable (the analysis was performed with available data from Study 1 and Study 2; $N=333$ ). The mean scores of gelotophobia of the six groups ranged from 1.80 to 1.91 and did not differ significantly from each other $(F(5,332)=.36, p=.90)$.

To answer the main question on the relation between gelotophobia and intelligence, the scores of the respective scales were correlated. For controlling the effects of age and sex on certain abilities, we computed partial-correlations as well. The same analyses were performed for the self-estimated intelligence. Additionally, partial correlations were computed between gelotophobia and intelligence controlling for self-estimated intelligence and the correlations to self-estimated intelligence controlling for psychometrically measured intelligence. Study 2 also allowed correlating the self-estimations of abilities with gelotophobia. The psychometrically measured abilities and the self-estimations of abilities correlated in the expected range. The correlation coefficients were $.19(p<.05)$ for verbal, .50 for numeric $(p<.01), .16(p<.05)$ for spatial intelligence and $.38(p<.01)$ for the total score of these tests with the total score of all 
Table 2. Correlations among psychometrically measured, self-estimated intelligence, and a difference score and gelotophobia

\begin{tabular}{|c|c|c|c|c|c|c|c|c|c|}
\hline & \multicolumn{2}{|c|}{$\begin{array}{c}\text { Psychometric } \\
\text { (Study 1) }\end{array}$} & \multicolumn{2}{|c|}{$\begin{array}{c}\text { Psychometric } \\
\text { (Study 2) }\end{array}$} & \multicolumn{2}{|c|}{ Self-estimated } & \multicolumn{3}{|c|}{$P M-S E$} \\
\hline & $\mathrm{r}$ & $\mathrm{r}(\mathrm{sex}$, age $)$ & $\mathrm{r}$ & $\mathrm{r}($ sex,age $)$ & $\mathrm{r}$ & r(sex,age) & $\mathrm{r}(\mathrm{IQ})$ & $\mathrm{r}(\mathrm{SE})$ & $r($ Diff $)$ \\
\hline rrt & -.05 & -.11 & -.05 & -.06 & $-.16^{*}$ & -.1 & -.14 & -.02 & .08 \\
\hline Numer & -.05 & -.13 & -.02 & $-.17 *$ & -.02 & - & .01 & -.02 & -.02 \\
\hline Spatial & -.06 & -.09 & -.04 & -.12 & -.07 & - & -.07 & -.03 & .03 \\
\hline Memo & $-.19 *$ & $-.24 * *$ & -.11 & - . & $-.16^{*}$ & - & -.14 & -.08 & .04 \\
\hline 10 & -.07 & -.05 & -.06 & $-.17^{*}$ & $-.18^{*}$ & $-.27 * *$ & $-.20 * *$ & .03 & $.21^{*}$ \\
\hline Reasoning & -.06 & -.14 & -.09 & $-.18^{*}$ & -.12 & -.18 & -.10 & -.05 & .06 \\
\hline Vocabulary & .03 & -.13 & $-.16^{*}$ & $-.21^{*}$ & $-.22 * *$ & $-.22 * *$ & $-.19 *$ & -.09 & $.21^{* *}$ \\
\hline Attention & -.02 & -.10 & .07 & .01 & $-.15^{*}$ & $-.18 *$ & $-.16^{*}$ & .09 & $.17 *$ \\
\hline
\end{tabular}

Study 1: $N=168$ and $N=136$ for the partial correlation; Study 2: $N=151-177 ; N=150$ for partial correlation (psychometric) and $N=164$ for partial correlation (self-estimated); $r($ sex,age $)=$ partial correlation controlling for sex and age; $r(\mathrm{IQ})=$ correlation with psychometrically measured intelligence controlled for self-estimated intelligence; $r(\mathrm{SE})=$ correlation with self-estimated intelligence controlled for psychometrically measured intelligence; $r($ Diff $)=$ difference score of psychometrically measured and self-estimated intelligence. Total $=$ total score of verbal, numeric, and spatial intelligence for psychometrically measured intelligence and a global score of all estimations for the self-estimated intelligence. ${ }^{*} p<.05 ; * * p<.01$

self-estimations, $.23(p<.05)$ for memory, $.38(p<.01)$ for reasoning, and $.29(p<.01)$ for the vocabulary. Only one non-significant correlation was found; i.e., for attention $(r=.13)$. Finally, differences for (standardized) means in psychometrically measured and self-estimated intelligence were computed. This variable was used as an indicator for high/low selfestimations of the own ability (i.e., the higher the positive correlations the lower the self-estimations). Results are shown in Table 2.

Table 2 shows that gelotophobia and intelligence were not correlated. The only exceptions were significant correlations between lower memory abilities (Study 1) and lower vocabulary (Study 2) and higher expressions of gelotophobia. However, the results were virtually identical after controlling for age and gender in Study 1. Partial correlations led to negative relations between gelotophobia and memory (Study 1) and numeric intelligence, the total score of cognitive abilities, reasoning and vocabulary (Study 2; all between $r=-.17$ and $r=-.24, p<.05$ ). Controlling for self-estimated intelligence led to a negative relation of the total score to gelotophobia. Moreover, controlling for psychometrically measured intelligence led to zero correlations in all tests. 
Self-estimations of their own intelligence were lower for gelotophobes in verbal intelligence, memory, attention, and the vocabulary $(r=-.15$ to $r=-.22, p<.05$ ). Also, the total score (i.e., composed of verbal, numeric, and spatial intelligence) was negatively related to gelotophobia $(r=-.18, p<.01)$. While partial correlations controlling for age led to the same results, controlling for sex led to significantly negative relations to the self-estimated spatial intelligence and reasoning $(p<.05)$. Thus, the self-estimations of one's own abilities were generally lower among gelotophobes. The difference scores (psychometrically measured intelligence vs. self-estimations) indicated that gelotophobes, in particular had lower self-estimations regarding their general intelligence (i.e., total score), vocabulary, and attention.

Comparisons of the actual self-estimations in groups of nongelotophobes, borderline, slight, as compared to pronounced gelotophobes with the highest possible self-estimations also showed this tendency of lower self-estimations among gelotophobes. In this analysis a score of $100 \%$ would indicate that the observed self-estimations and the highest possible self-estimations were identical (i.e., all participants scored on the high end of the positive pole). While the median of these selfestimations was $64.19 \%$ in the group of non-gelotophobes (in relation to the highest possible scores) the medians decreased in the other groups; $59.67 \%, 58.50 \%$, and $49.13 \%$ for participants with borderline, slight, and pronounced expressions of gelotophobia. The lowest relation was found for numeric intelligence in the group of pronounced gelotophobes $(35.59 \%)$ and the highest was found for vocabulary in the group of nongelotophobes $(71.80 \%)$. Overall, the results showed that pronounced gelotophobes differed most from the highest possible self-estimations.

\section{Do gelotophobes underestimate their abilities?}

The previous analyses have shown that gelotophobes have lower selfestimations of their own abilities. However, it is also interesting to know whether or not they possibly have a realistic view of their abilities and therefore lower self-estimations, in contrast to the non-gelotophobes who probably over-estimate their abilities. Therefore, we regressed sex and age on each of the psychometrically measured and self-estimated abilities and computed standardized residual scores. Afterwards, these scores were transformed to IQ-scores $(M=100, S D=15)$. Figure 1 shows the 


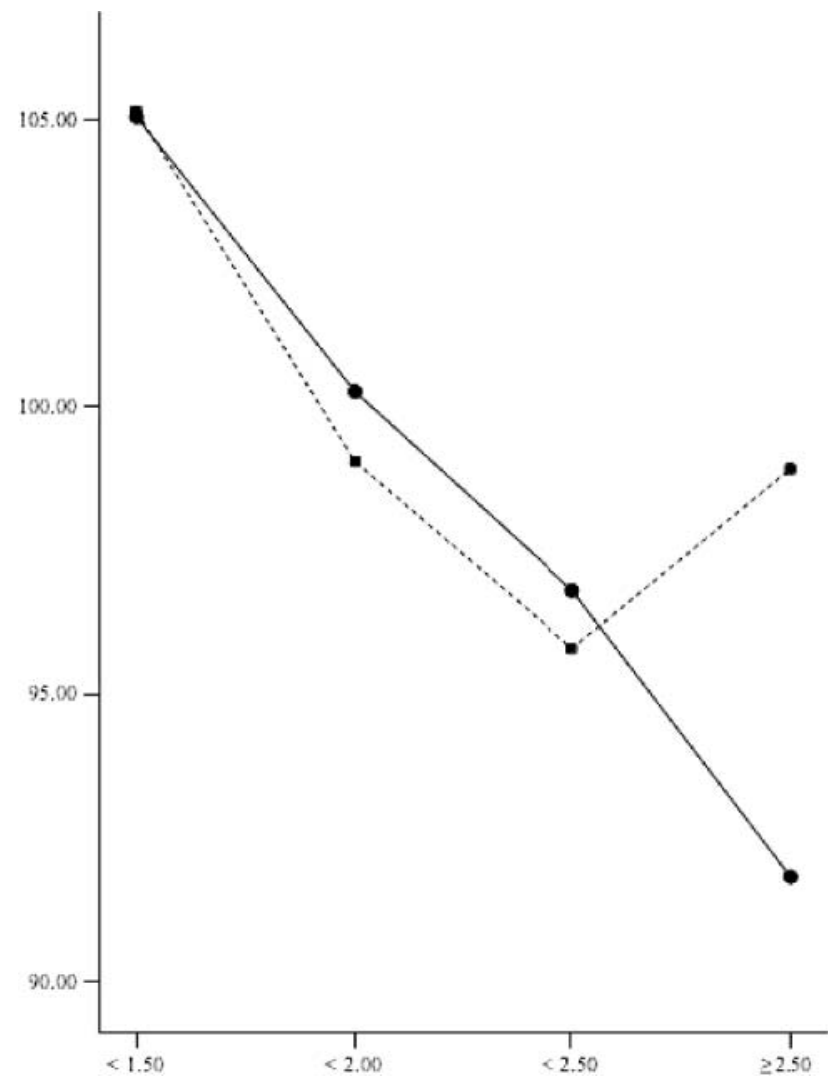

Figure 1. IQ-scores for the total scores of psychometrically measured (dotted line) and self-estimated (full line) intelligence (total scores, controlled for sex and age) for gelotophobes (i.e., mean score $\geq 2.50 ; 16 \leq N \geq 17$ ) and three groups of non-gelotophobes (44 $\leq N \geq 53$ )

distribution of these scores for groups of non-gelotophobes (mean score $<1.50$ and $<2.00$ ), borderline gelotophobes (means between 2.00 and 2.50), and gelotophobes (means $>2.50$ ).

Figure 1 shows that the transformed IQ-scores for psychometrically measured and self-estimated abilities were highly similar in all three groups of non-gelotophobes (including a borderline-category with mean scores ranging from 2.00 to $<2.50$ ). However, the gelotophobes' selfestimations of their own abilities were lower than the psychometrically measured scores. They underestimated their intelligence by slightly more 
than one third of the standard deviation; i.e., 6 IQ-points (this is a difference of approximately 18 percent-ranks). ANOVAs were computed with the total scores for psychometrically measured and self-estimated intelligence as dependent variables and the cut-off scores used in Figure 1 as classification variable (4 groups). In both cases, significant main effects were found (psychometric $F(3,160)=3.56, p<.05$; self-estimated $F(3,168)=3.76, p<.05)$. For the psychometrical intelligence, the lowest gelotophobia scorers (mean scores $<1.50$ ) had significantly higher averaged IQ-scores $(M=104.50)$ than the gelotophobes $(M=92.90)$. The lowest scorers also had significantly higher self-estimations $(M=105.15)$ than participants with mean scores between 1.50 and $1.99(M=99.04$, $p<.05)$ and the borderline gelotophobes $(M=95.79, p<.01$; the latter two groups were not different from each other). Though the mean score for the gelotophobes was lower as well $(M=98.92)$, it did not differ significantly from the lowest scorers in gelotophobia. However, this might be linked to a loss of statistical power due to the low number of persons in this group.

\subsection{Comparisons based on normative data}

For examining whether or not gelotophobes truly underestimate their abilities, all raw scores were transformed to IQ-scores based on the mean scores and standard deviations of the whole group. It was also important to know whether there were differences in the psychometric test scores with respect to the normative samples. Therefore, the raw scores for verbal intelligence were transformed to the IQ-scores ${ }^{1}$ based on the norm values (split by age) for the total group. The whole sample from studies 1 and 2 was used for the following analyses ( $N=323$ participants). They exceeded the expected IQ-scores $(=100)$ with a mean of 108.65 $(\mathrm{SD}=13.82)$. The IQs ranged from 64 to 148 . Next, to see whether non-gelotophobes and gelotophobes differed in their intelligence, an ANOVA was performed with groups of non-gelotophobes $(I Q=109.68)$, borderline gelotophobes ( $\mathrm{IQ}=107.48)$, and slight $(\mathrm{IQ}=105.71)$ and pronounced gelotophobes (including extreme cases; IQ $=105.19$ ) as classification variable and the IQ as dependent variable. The scores were not significantly different from each other $(F(3,322)=1.24$, n.s.). Therefore, gelotophobes did not differ from non-gelotophobes if the analysis was computed with IQ-scores based on the normative data of the I-S-T 2000 $\mathrm{R}$ (for verbal intelligence ${ }^{2}$ ). However, it should be mentioned that the 
IQ-scores for the group of pronounced/extreme gelotophobes were lowest in all analyses. Therefore, it may be that mean-score differences will be revealed in a sample with more participants in this category as the low number of group members $(n=12)$ might have compromised the results of the mean comparisons.

\section{Discussion}

The present study was aimed at an examination of the relationship between gelotophobia and psychometrically measured and self-estimated intelligence. As expected, gelotophobes did not score lower in psychometric ability tests. In Study 1 there was a negative correlation to memory and in Study 2 to vocabulary. However, the correlations were low in size and accounted for roughly $4 \%$ of the variance. Taken together, the two studies show that ability and gelotophobia exist independently from each other. However, the results indicate that the lowest scores in gelotophobia (mean scores $>1.50$ in the GELOPH) are related to the highest IQ-scores and the highest self-estimations of one's own abilities. Therefore, the lowest scorers in the fear of being laughed at in the present sample outperformed the others in both the psychometrically measured and the self-estimated general intelligence. However, a larger sample size might be needed in future studies as the numbers of participants in the category with extreme gelotophobia was low and therefore might have negated differences in mean score comparisons. As a tendency, the mean scores of higher scoring gelotophobes were lowest for almost all ability tests.

One of the ideas in the introductory section was that persons with low intellectual abilities fear being laughed at because they experience their own inferiorities compared to others often in their daily lives. This does not seem to be the case for gelotophobes, as they did not differ from non-gelotophobes regarding their abilities. However, this sample might not be best suited for finally rejecting this hypothesis. The mean score for verbal intelligence was 109 for the samples (an IQ $=100$ would have been expected) and therefore probably did not have enough low(er) scoring participants in the sample. Therefore, it is unclear whether low intellectual ability might be a reason to develop gelotophobia (i.e., the lack of ability to see one is not being laughed at). A different sample including more low(er) scorers would be needed to answer that question.

Even if gelotophobes do not differ from others regarding their own abilities, the more important practical question seems to be related to the 
self-estimations of their own abilities. These results suggest that gelotophobes tend to have low self-estimations regarding their abilities and especially their general intelligence, vocabulary, and attention. They do not only have lower self-estimations of their own abilities but they underestimate their true abilities (psychometrically measured) by 6 IQ-points (i.e. a difference of approx. 18 percent-ranks).

The feelings of inferiority, lower self-estimation, and underestimation of their own abilities (be it intelligence, be it humor creation; see Ruch et al. this issue) seems to be a general pattern among gelotophobes. This might be of relevance for the development of treatments for gelotophobia. Helping gelotophobes to achieve a more realistic self-estimation may be a key-strategy for lasting improvements. However, this is at the level of speculations and needs to be tested empirically in more detail. Also, different explanations of the results might apply. For example, the lower estimations of one's own abilities might be due to the modesty of gelotophobes (see Proyer and Ruch this issue). In that case, they would know their abilities but provide lower self-estimations for avoiding appearing overbearing to others (i.e., another source for being laughed at).

As a future research direction, it is important to repeat the study including measures for gelotophilia (the joy of being laughed at) and katagelasticism (the joy of laughing at others; see Ruch and Proyer this issue). It will be of interest whether or not gelotophiles and katagelasticists differ from non-gelotophiles and non-katagelasticists in their intelligence and their self-estimated abilities or whether they differ from high scoring gelotophobes. In Ruch et al. (this issue) it was shown that gelotophiles and katagelasticists do not have higher humor creation abilities. Nevertheless, one might expect that katagelasticists assume they are more intelligent than others, and, therefore, enjoy mocking and ridiculing them. However, the relation to intelligence needs to be further examined empirically.

University of Zurich

\section{Notes}

Correspondence address: r.proyer@psychologie.uzh.ch

* The study was supported by a research grant from the University of Zurich (FK56231101) and the Suzanne and Hans Biäsch Foundation (Zurich, Switzerland). The authors are grateful to Claudia Buschor, Fabian Gander, Iwana Städeli, and Tobias Wyss who helped with the data collection and the data input. 
1. The handbook provides Z-scores that were transformed to IQ-scores for the further analyses.

2. The same results were found by performing ANOVAs with transformed IQ-scores for numeric $(F(3,320)=0.23$, n.s. $)$ and spatial intelligence $(F(3,327)=2.38$, n.s. $)$.

\section{References}

Ackerman, Philipp, L., and Eric D. Heggestad

1997 Intelligence, personality, and interests: Evidence for overlapping traits. Psychological Bulletin 21 (2), 219-245.

Amthauer, Rudolf, Burkhard Brocke, Detlev Liepmann, and André Beauducel

2001 Intelligenz-Struktur-Test $2000 R(I-S-T 2000 R)$ [Intelligence Structure

Brickenkamp, Rolf Test]. Göttingen: Hogrefe.

1994 Test d2 Aufmerksamkeits-Belastungs-Test [d2 Test of attention]. Göttingen: Hogrefe.

Chamorro-Premuzic, Tomas, Adrian Furnham, and Joanna Moutafi

2004 The relationship between estimated and psychometric personality and intelligence scores. Journal of Research in Personality 38, 505-513.

Chamorro-Premuzic, Tomas, Joanna Moutafi, and Adrian Furnham

2005 The relationship between personality traits, subjectively-assessed and fluid intelligence. Personality and Individual Differences 38, 1517-1528.

Cooper, Colin

2002 Individual Differences, 2nd ed. London: Arnold.

Eysenck, Hans J.

1994 Personality and intelligence: psychometric and experimental approaches. In Sternberg, Robert J., and Patricia Ruzgis (eds.), Personality and Intelligence. Cambridge University Press, 3-31.

Furnham, Adrian

2001 Self-estimates of intelligence: culture and gender difference in self and other estimates of both general $(\mathrm{g})$ and multiple intelligences. Personality and Individual Differences 31 (8), 1381-1405.

Furnham, Adrian, and Tomas Chamorro-Premuzic

2004 Estimating one's own personality and intelligence scores. British Journal of Psychology 95, 149-160.

Hopko, Derek R., Julie A. Crittendon, Erick Grant, and Sarah A. Wilson

2005 The impact of anxiety on performance IQ. Anxiety, Stress, and Coping 18 (1), 17-35.

Köhler, Gabriele, and Willibald Ruch

1993 The Cartoon Punch line Production Test - CPPT. Unpublished manuscript, University of Düsseldorf, Department of Psychology, Düsseldorf, Germany.

1996 Sources of variance in current sense of humor inventories: How much substance, how much method variance? Humor: International Journal of Humor Research 9 (3/4), 363-397.

Mabe, Paul A. and Stephen G. West

1982 Validity of self-evaluation of ability: A review and meta-analysis. Journal of Applied Psychology 67 (3), 280-296. 
Meijer, Joost and Ron Oostdam

2007 Test anxiety and intelligence testing: A closer examination of the stage-fright hypothesis and the influence of stressful instruction. Anxiety, Stress, and Coping 20 (1), 77-91.

Moutafi, Joanna, Adrian Furnham, and Ioannis Tsaousis

2006 Is the relationship between intelligence and trait Neuroticism mediated by test anxiety? Personality and Individual Differences 40 (3), 587-597.

Perkins, Adam A., and Philipp J. Corr

2006 Cognitive ability as a buffer to neuroticism: Churchill's secret weapon? Personality and Individual Differences 40 (1), 39-51.

Platt, Tracey

2008 Emotional responses to ridicule and teasing: Should gelotophobes react differently? Humor: International Journal of Humor Research 21 (2), 105-128.

Proyer, René T. and Willibald Ruch

2008 Measure for self-estimated intelligence (MSEI). Unpublished questionnaire, University of Zurich, Switzerland.

Raven, John C., Raven John, and John H. Court

1998 Standard Progressive Matrices (Handbook; Editors of the German form: Stephan Bulheller and Hartmut O. Häcker). Frankfurt: Swets Test Services.

Ruch, Willibald and René T. Proyer

2008a Who is gelotophobic? Assessment criteria for the fear of being laughed at. Swiss Journal of Psychology 67 (1), 19-27.

2008b The fear of being laughed at: Individual and group differences in gelotophobia. Humor: International Journal of Humor Research 21 (1), 47-67.

Schmidt, Karl-Heinz, and Peter Metzler

1992 Wortschatztest (WST) [Vocabulary test]. Weinheim: Beltz. 